\title{
THE INHIBITORY EFFECT OF MK886 ON THE INFLAMMATORY PROCESS CAUSED BY Paracoccidioidomycosis brasiliensis INFECTION IN GENETICALLY SELECTED MICE
}

Thesis: H. A. Balderramas submitted this thesis for his Masters in Tropical Diseases at the Botucatu Medical School, São Paulo State University, UNESP, Botucatu, São Paulo State, Brazil, 2008.

Advisor: Professor Silvio Luis de Oliveira

ABSTRACT: Leukotrienes are classic inflammatory response mediators considered chemotactic agents and microbicidal activity regulators in cells of the innate immune system, playing a protective role against different infectious agents. In this study, we investigated the involvement of leukotrienes in the course of murine paracoccidioidomycosis based on the following immunologic parameters: cell influx, mieloperoxydase activity, NO production, cytokine production, and fungal recovery in lungs of mice selected according to the intensity of their low (AIRmin) and high (AIRmax) acute inflammatory response. Infection by $P$. brasiliensis induced considerable production of IL-6, IL-10, IFN-gamma and TNF-alpha cytokines, and led to cell recruitment, as well as NO production in lungs at different study periods. In animals treated with MK886, a leukotriene biosynthesis inhibitor, IFN-gamma, IL-6 and TNF-alpha production was lower, while neutrophil influx and NO production decreased. These results may explain the higher fungal load in lungs of animals in which leukotriene synthesis was inhibited, suggesting that leukotrienes have a possible protective role in experimental paracoccidioidomycosis. AIRmax animals had lower fungal load in comparison with AIRmin ones, which can be related to the AIR phenotype regarding neutrophil migration, besides lower production of NO and pro-inflammatory cytokines. Thus, mice presenting AIRmax background are more resistant to infection by $P$. brasiliensis.

KEY WORDS: leukotrienes, Paracoccidioides brasiliensis, neutrophils, innate immune response.

\section{CORRESPONDENCE TO:}

HELANDERSON DE ALMEIDA BALDERRAMAS, Departamento de Microbiologia e Imunologia, Instituto de Biociências de Botucatu, UNESP, Botucatu, SP, 18618-970, Brasil. Phone: +55 1438116058 or +55 143811 6240. Email:

helbalderramas@yahoo.com.br. 